\title{
Lyapunov Pairs in Semilinear Differential Problems with State-Dependent Impulses
}

\author{
I. Benedetti ${ }^{1}$ - T. Cardinali $^{1}$ - G. Gabor ${ }^{2}$ (D) P. Rubbioni ${ }^{1}$
}

Received: 26 October 2017 / Accepted: 21 June 2018 / Published online: 6 July 2018

(C) The Author(s) 2018

\begin{abstract}
The paper deals with semilinear differential inclusions with state-dependent impulses in Banach spaces. Defining a suitable Banach space in which all the solutions can be embedded we prove the first existence result for at least one global mild solution of the problem considered. Then we characterize this result by means of a new definition of Lyapunov pairs. To this aim we give sufficient conditions for the existence of Lyapunov pairs in terms of a new concept of contingent derivative.
\end{abstract}

Keywords Differential inclusions · State-dependent impulses · Lyapunov pairs · Contingent derivative

Mathematics Subject Classification (2010) Primary: 34A60 - Secondary: 34A37, 37B25

\section{Introduction}

Impulsive differential equations or inclusions describe phenomena characterized by the fact that the model parameters are subject to short-term perturbations in time. For instance, in

\section{G. Gabor}

ggabor@mat.umk.pl

I. Benedetti

irene.benedetti@unipg.it

T. Cardinali

tiziana.cardinali@unipg.it

P. Rubbioni

paola.rubbioni@unipg.it

1 Department of Mathematics and Computer Science, University of Perugia, Perugia, Italy

2 Faculty of Mathematics and Computer Science, Nicolaus Copernicus University in Torun, Torun, Poland 
the periodic treatment of some diseases, impulses may correspond to administration of a drug treatment; in environmental sciences, impulses may correspond to seasonal changes or harvesting; in economics, impulses may correspond to abrupt changes of prices. For a bibliography on the theory of impulsive differential equations one can see, for instance, the monographs $[4,25]$ and for more recent results on impulsive differential inclusions we refer the interested reader to some papers and monographs of the last decade: [7, 8, 18, 19]. All the cited papers deal with fixed moments of impulses, but in fact as well as fixed beforehand the moments of impulses can be chosen in various ways, for instance, randomly, or determined by the state of a system. Existence results for several kind of impulsive differential inclusions with variable times of impulses in a finite dimensional framework are contained in $[5,6,16,17,20]$. Some interesting results on classical solutions to single-valued impulsive problems in abstract spaces (with state-dependent impulses) can be found in, e.g., [21]. As far as we know this work is the first attempt to solve the problem of the existence of mild solutions for semilinear differential inclusions with impulses at variable times in Banach spaces.

In particular, given $a>0$ we consider the following semilinear differential inclusion with state-dependent impulses:

$$
(I P) \begin{cases}y^{\prime}(t) \in A y(t)+G(t, y(t)), & t \in[0, a], t \neq \tau_{j}(y(t)), j=1, \cdots, m \\ y\left(t^{+}\right)=y(t)+I_{j}(y(t)), & t \in[0, a], t=\tau_{j}(y(t)), j=1, \cdots, m \\ y(0)=y_{0} \in E, & \end{cases}
$$

where $E$ is a Banach space endowed with the norm $\|\cdot\| ; A: D(A) \subseteq E \rightarrow E$ is a linear operator satisfying

(A) $\quad A$ is the infinitesimal generator of a $C_{0}$-semigroup $\{U(t)\}_{t \geq 0}$;

$G:[0, a] \times E \multimap E$ is a given multivalued map; $\tau_{j}: E \rightarrow \mathbb{R}$ and $I_{j}: E \rightarrow E$, $j=1, \cdots, m$, are given maps; $y\left(t^{+}\right)=\lim _{s \rightarrow t^{+}} y(s)$.

Problems with state-dependent impulses are more suitable to describe real-life phenomena, but more complicated from a theoretical point of view. Indeed, while for problems with fixed moments of impulses (i.e., with $\tau_{j}$ constant functions for any $j=1, \ldots, m$ ) several methods from continuous problems can be adopted, this is not the case for state-dependent impulses. One of the main difficulties is to find a suitable function space, where all solutions could be embedded. Recently, in [20], where $E=\mathbb{R}^{n}$, such a function space (which is a Banach space) is introduced and, under suitable assumptions, all solutions are interpreted as elements of this space (for non-metrizable space see, e.g., [3]).

In this paper, we extend this definition from a finite-dimensional setting to an infinite one obtaining the first existence result for at least one global mild solution of the problem $(I P)$ (see Theorem 2.4). Then we characterize this first result by means of a new definition of a Lyapunov pair (called equi-Lyapunov pair) based on the definition of a Lyapunov pair given in [10]. Roughly speaking we prove the existence of at least one mild solution in the case that the barrier $\tau_{j}$ is the first component of the equi-Lyapunov pair (see Theorem 3.6).

We recall that Cârjă in [10] considers the following autonomous Cauchy problem

$$
(A P)_{\xi}\left\{\begin{array}{l}
y^{\prime}(t) \in A y(t)+G(y(t)), \text { for a.e. } t \in \mathbb{R} \\
y(0)=\xi \in E,
\end{array}\right.
$$

where $A: D(A) \subseteq E \rightarrow E$ is an operator and $G: M \multimap E$ is a multimap, with $M$ a nonempty set in the Banach space $E$. According to [10], given $V, p: M \rightarrow[-\infty,+\infty]$, two maps, the couple $(V, p)$ is said to be a Lyapunov pair for the inclusion $y^{\prime} \in A y+G(y)$ if, for every $\xi \in D(V)=\{x \in E: V(x)<+\infty\}$ there exists $T>0$ and a mild solution 
$y:[0, T] \rightarrow M$ to the autonomous multivalued semilinear Cauchy problem $(A P)_{\xi}$, the map $t \mapsto p(y(t))$ is integrable on $[0, T]$ and

$$
V(y(t))+\int_{0}^{t} p(y(s)) d s \leq V(\xi), \text { for all } t \in[0, T] .
$$

In the case where $E$ is an Hilbert space, $G$ is single-valued and Lipschitz continuous, and $A$ is a not necessarily linear operator, Kocan and Soravia in [24] characterized the Lyapunov pairs using the partial differential inequality

$$
\langle-A(x), D V(x)\rangle+\langle G(x), D V(x)\rangle \geq p(x), \text { for every } x \in D(A)
$$

whose solutions $V$ are meant in the viscosity sense.

Recently, in the same setting of hypotheses as in [24], but with the operator $A$ possibly multivalued, in [1,2], Adly et al. provide primal and dual criteria for weakly lower semicontinuous Lyapunov pairs, explicitly formulated by means of the proximal and basic subdifferentials of the involved functions.

In [12] Cârjă and Motreanu, considering an appropriate contingent derivative, obtain a different characterization of Lyapunov pairs on Hilbert spaces, with $A$ a linear operator and $G$ a locally Lipschitz mapping. Their approach is based on viability results and uses the contingent derivative associated to the operator $A$ defined as follows.

Definition 1.1 Let $A: D(A) \subseteq E \rightarrow E$ be an operator satisfying (A), $V: E \rightarrow$ ] $-\infty,+\infty]$ be a map with a non-empty effective domain $D(V)=\{x \in E: V(x)<+\infty\}$. The A-contingent derivative $\underline{D}^{A} V(x)(u)$ of $V$ at $x \in D(V)$ in the direction $u \in E$ is defined by

$$
\underline{D}^{A} V(x)(u)=\liminf _{\substack{h \downarrow 0 \\ w \rightarrow 0_{E}}} \frac{1}{h}\left[V\left(U(h) x+\int_{0}^{h} U(h-s) u d s+h w\right)-V(x)\right] .
$$

Later the same authors in [13] extend this result to arbitrary Banach spaces and to $m$ dissipative possibly multivalued operators $A$. In [11] Cârjă and Lazu obtain an analogous characterization assuming only continuity on $G$ but requiring that the semigroup generated by the $m$-dissipative operator is compact. Assuming that $A$ is a linear operator generating a compact semigroup, Cârjă in [10] extends the results obtained in the previous cited papers to the case of a multivalued map $F$. To this aim he introduces a new concept of contingent derivative suitable for inclusions. Precisely, given a nonempty bounded set $S \subset E$, he considers the set

$$
S_{L^{1}}=\left\{f \in L_{\text {loc }}^{1}\left(\mathbb{R}^{+} ; E\right): f(s) \in S \text { a.e. } s \in \mathbb{R}^{+}\right\},
$$

then the A-contingent derivative of $V$ at $x \in D(V)$ in the direction $S \subset E$ is defined as follows.

Definition 1.2 Let $A: D(A) \subseteq E \rightarrow E$ be an operator satisfying (A), $V: E \rightarrow]-$ $\infty,+\infty]$ be a map with non-empty effective domain $D(V)=\{x \in E: V(x)<+\infty\}$. The A-contingent derivative $\underline{D}^{A} V(x)(S)$ of $V$ at $x \in D(V)$ in the direction $S \subset E$ is defined by

$$
\underline{D}^{A} V(x)(S)=\liminf _{\substack{h \downarrow 0 \\ w \rightarrow 0_{E}}} \inf _{f \in S_{L^{1}}} \frac{1}{h}\left[V\left(U(h) x+\int_{0}^{h} U(h-s) f(s) d s+h w\right)-V(x)\right] .
$$

In this paper we introduce a new type of Lyapunov pair, not depending on a mild solution. 
Definition 1.3 Given two maps $V, p: E \rightarrow \mathbb{R}$, we say that $(V, p)$ is an equi-Lyapunov pair for the inclusion

$$
y^{\prime}(t) \in A y(t)+G(t, y(t)) \text {, for a.e. } t \in[0, a],
$$

if, for every $(\tau, \xi) \in[0, a[\times E$ and every mild solution $y:[\tau, a] \rightarrow M$ to the multivalued semilinear Cauchy problem

$$
(P)_{(\tau, \xi)}\left\{\begin{array}{l}
y^{\prime}(t) \in A y(t)+G(t, y(t)), \text { for a.e. } t \in[\tau, a] \\
y(\tau)=\xi
\end{array}\right.
$$

the map $t \mapsto p(y(t))$ is integrable on $[\tau, a]$ and

$$
V(y(t))+\int_{\tau}^{t} p(y(s)) d s \leq V(\xi), \text { for all } t \in[\tau, a] .
$$

Following the approach described in [10], we give sufficient conditions for the existence of equi-Lyapunov pairs in terms of a new concept of $A$-contingent derivative defined as follows.

Definition 1.4 Let $A: D(A) \subseteq E \rightarrow E$ be an operator satisfying (A), $V: E \rightarrow]-$ $\infty,+\infty]$ be a map with non-empty effective domain $D(V)=\{x \in E: V(x)<+\infty\}$. The strong-A-contingent derivative of $V$ at $x \in D(V)$ in the direction $f \in S_{L^{1}}$ is defined by

$$
\underline{D}^{A} V(x)(f)=\liminf _{\substack{h \downarrow 0 \\ w \rightarrow 0_{E}}} \frac{1}{h}\left[V\left(U(h) x+\int_{0}^{h} U(h-s) f(s) d s+h w\right)-V(x)\right] .
$$

We point out the fact that unlike all the cited results we consider a non-autonomous differential inclusion on arbitrary Banach spaces with $A$ a linear operator generating a non necessarily compact semigroup.

Actually, to obtain existence results for mild solutions of the problem $(I P)$ it is sufficient to consider a weaker definition of equi-Lyapunov pair, namely:

Definition 1.5 Given two maps $V, p: E \rightarrow \mathbb{R}$, we say that $(V, p)$ is an equi-Lyapunov pair for problem $(P)_{(\tau, \xi)}$ if, for every mild solution $y:[\tau, a] \rightarrow M$ to $(P)_{(\tau, \xi)}$, the map $t \mapsto p(y(t))$ is integrable on $[\tau, a]$ and

$$
V(y(t))+\int_{\tau}^{t} p(y(s)) d s \leq V(\xi), \text { for all } t \in[\tau, a] .
$$

Using this definition it is possible to consider $p: E \rightarrow \mathbb{R}$ depending on the initial values $(\tau, \xi)$ of the Cauchy problem.

The paper is organized as follows: in Section 2 we prove an existence result for the impulsive problem $(I P)$; in Section 3, after a preliminary study of the autonomous case (see Section 3.1), we give sufficient conditions to have an equi-Lyapunov pair for the differential inclusion (2) (see Section 3.2) and then we obtain another existence result for (IP) via equi-Lyapunov pairs defined as in Definition 1.5 (see Section 3.3); in Section 4 we give the concluding remarks; finally, for the reader's convenience in the Appendix we recall some definitions and results the proofs are based on. 


\section{Semilinear Differential Problem with State Dependent Impulses}

In this Section we consider the problem $(I P)$ under the following assumptions.

(H1) for every $j=1, \ldots, m$, the map $\tau_{j}: E \rightarrow \mathbb{R}$ is a continuous function; moreover for every $x \in E$ it holds that

(H1.1) $0<\tau_{j}(x)<\tau_{j+1}(x)<a, j=1, \ldots, m-1$;

(H1.2) $\tau_{j}\left(x+I_{j}(x)\right) \leq \tau_{j}(x)<\tau_{j+1}\left(x+I_{j}(x)\right), j=1, \ldots, m-1$;

(H1.3) $\tau_{m}\left(x+I_{m}(x)\right) \leq \tau_{m}(x)$.

We search for the existence of mild solutions of problem $(I P)$ in the space

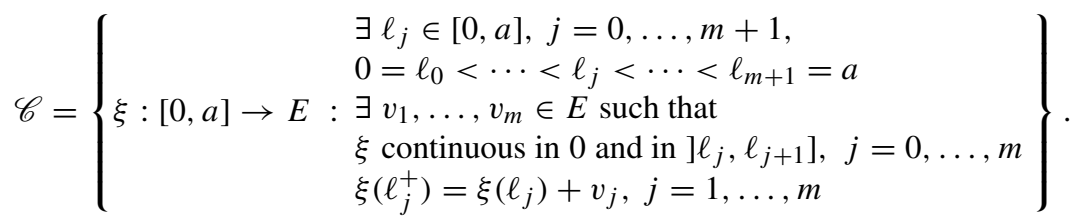

A mild solution of the problem $(I P)$ is a map belonging to the space $\mathscr{C}$ such that it meets exactly once each hypersurface $\Sigma_{j}=\left\{(t, x) \in[0, a] \times E: t-\tau_{j}(x)=0\right\}$, for every $j=1, \ldots, m$. More precisely, we give the following definition.

Definition 2.1 A mild solution of problem $(I P)$ is a map $y \in \mathscr{C}$ such that for every $j=$ $1, \ldots, m$ there exist a unique $\left.t_{j} \in\right] 0, a$ [ with $\tau_{j}\left(y\left(t_{j}\right)\right)=t_{j}$ and a map $g \in L^{1}([0, a] ; E)$, $g(s) \in G(s, y(s))$ for a.e. $s \in[0, a]$, such that

$$
y(t)=U(t) y_{0}+\sum_{0<t_{j}<t} U\left(t-t_{j}\right) I_{j}\left(y\left(t_{j}\right)\right)+\int_{0}^{t} U(t-s) g(s) d s, t \in[0, a] .
$$

To prove the existence of at least one mild solution of the problem (IP) we assume the following hypotheses on the multimap $G:[0, a] \times E \multimap E$ :

(G1) for every $(t, x) \in[0, a] \times E, G(t, x)$ is convex and compact;

(G2) for every $x \in E$, the multimap $G(\cdot, x)$ admits a strongly measurable selector, i.e. there exists a strongly measurable function $q:[0, a] \rightarrow E$ such that $q(t) \in G(t, x)$ for a.a. $t \in[0, a]$

(G3) for every $t \in[0, a]$, the multimap $G(t, \cdot)$ is upper semicontinuous;

(G4) there exists $\alpha \in L_{+}^{1}([0, a])$ such that

$$
\|G(t, x)\| \leq \alpha(t)(1+\|x\|), \text { for a.e. } t \in[0, a] \text { and every } x \in E ;
$$

(G5) there exists $\beta \in L_{+}^{1}([0, a])$ such that

$$
\chi(G(t, D)) \leq \beta(t) \chi(D), \text { for every bounded set } D \subset E \text {, a.e. } t \in[0, a],
$$

where $\chi$ is the Hausdorff measure of noncompactness.

Remark 2.2 Notice that, under the above assumptions on the multimap $G$, for every $(\tau, \xi) \in$ $[0, a[\times E$ the Cauchy problem

$$
(P)_{(\tau, \xi)}\left\{\begin{array}{l}
y^{\prime}(t) \in A y(t)+G(t, y(t)), \text { for a.e. } t \in[\tau, a] \\
y(\tau)=\xi
\end{array}\right.
$$


admits at least one global mild solution by Theorem 4 in [9]. Therefore

$$
\mathcal{S}_{(\tau, \xi)}:=\left\{y:[\tau, a] \rightarrow E: y \text { is a global mild solution of }(P)_{(\tau, \xi)}\right\} \neq \emptyset .
$$

Moreover, we need the following viability-type condition:

(H2) for every $j=1, \ldots, m,(\tau, \xi) \in\left[0, a\left[\times E, y \in \mathcal{S}_{(\tau, \xi)}\right.\right.$, we have

$$
\left.\left.\tau_{j}(y(t))-t<\tau_{j}(\xi)-\tau, \text { for every } t \in\right] \tau, a\right] .
$$

Remark 2.3 Notice that, if the functions $\tau_{j}, j=1, \ldots, m$, are constant, then the problem $(I P)$ comes down to a problem with fixed moments of impulses. Clearly in this case property $(H 2)$ is trivially satisfied.

Theorem 2.4 Let $E$ be a Banach space, $A: D(A) \subseteq E \rightarrow E$ be a linear operator satisfying (A), $G:[0, a] \times E \multimap E$ be a multimap such that (G1)-(G5) hold, and $\tau_{j}: E \rightarrow$ $\mathbb{R}, j=1, \ldots, m$, be functions satisfying $(\mathrm{H} 1)-(\mathrm{H} 2)$. Then the problem $(I P)$ admits at least one mild solution on the whole interval $[0, a]$.

Proof The proof splits in four steps.

STEP 1. Let $y^{0} \in \mathcal{S}_{\left(0, y_{0}\right)}$ (cf. (4)), i.e. $y^{0}:[0, a] \rightarrow E$ is defined as

$$
y^{0}(t)=U(t) y_{0}+\int_{0}^{t} U(t-s) g^{0}(s) d s, t \in[0, a],
$$

with $g^{0} \in L^{1}([0, a] ; E), g^{0}(s) \in G\left(s, y^{0}(s)\right)$ a.e. $s \in[0, a]$.

The function $y^{0}:[0, a] \rightarrow E$ defined in (6) is such that there exists $\left.t_{1}^{0} \in\right] 0, a[$ such that $t_{1}^{0}=\tau_{1}\left(y^{0}\left(t_{1}^{0}\right)\right)$. Indeed, let us introduce the map $w_{1}^{0}:[0, a] \rightarrow \mathbb{R}$ defined as

$$
w_{1}^{0}(t)=\tau_{1}\left(y^{0}(t)\right)-t, t \in[0, a]
$$

notice that $w_{1}^{0}$ is continuous by the continuity of the maps $y^{0}$ and $\tau_{1}$; moreover, by hypothesis (H1.1) we have

$$
\begin{aligned}
& w_{1}^{0}(0)=\tau_{1}\left(y^{0}(0)\right)>0 \\
& w_{1}^{0}(a)=\tau_{1}\left(y^{0}(a)\right)-a<a-a=0 .
\end{aligned}
$$

Then, there exists at least one $\left.t_{1}^{0} \in\right] 0, a$ [ such that $w_{1}^{0}\left(t_{1}^{0}\right)=0$, i.e. $t_{1}^{0}=$ $\tau_{1}\left(y^{0}\left(t_{1}^{0}\right)\right)$.

By the continuity of the map $w_{1}^{0}$, without loss of generality (w.l.o.g.) we can assume that $t_{1}^{0}=\min \{t \in] 0, a\left[: w_{1}^{0}(t)=0\right\}$.

STEP 2. We set $y_{1}=y^{0}\left(t_{1}^{0}\right)+I_{1}\left(y^{0}\left(t_{1}^{0}\right)\right)$. By hypothesis (H1.2) it follows that

$$
\tau_{1}\left(y_{1}\right)-t_{1}^{0} \leq \tau_{1}\left(y^{0}\left(t_{1}^{0}\right)\right)-t_{1}^{0}=0,
$$

i.e. $\left(y_{1}, t_{1}^{0}\right) \in \operatorname{epi}\left(\tau_{1}\right)=\left\{(y, t) \in E \times[0, a]: \tau_{1}(y) \leq t\right\}$; hence the point $\left(t_{1}^{0}, y_{1}\right)$ is on the right or belongs to the barrier $\Sigma_{1}$. Further, it is on the left of the barrier $\Sigma_{2}$, i.e. $\left(y_{1}, t_{1}^{0}\right)$ belongs to the set $\left\{(y, t) \in E \times[0, a]: \tau_{2}(y)>t\right\}$; indeed, by (H1.2) we have

$$
\tau_{2}\left(y_{1}\right)>\tau_{1}\left(y^{0}\left(t_{1}^{0}\right)\right)=t_{1}^{0} .
$$


We consider now a map $y^{1} \in \mathcal{S}_{\left(t_{1}^{0}, y_{1}\right)}$, i.e. $y^{1}:\left[t_{1}^{0}, a\right] \rightarrow E$ such that

$$
y^{1}(t)=U\left(t-t_{1}^{0}\right) y_{1}+\int_{t_{1}^{0}}^{t} U(t-s) g^{1}(s) d s, t \in\left[t_{1}^{0}, a\right]
$$

with $g^{1} \in L^{1}\left(\left[t_{1}^{0}, a\right] ; E\right), g^{1}(s) \in G\left(s, y^{1}(s)\right)$ for a.e. $s \in\left[t_{1}^{0}, a\right]$.

By conditions (H2) and (H1.2) we have

$$
\tau_{1}\left(y^{1}(t)\right)-t<\tau_{1}\left(y^{1}\left(t_{1}^{0}\right)\right)-t_{1}^{0}=\tau_{1}\left(y_{1}\right)-t_{1}^{0} \leq \tau_{1}\left(y^{0}\left(t_{1}^{0}\right)\right)-t_{1}^{0}=0,
$$

for every $\left.t \in] t_{1}^{0}, a\right]$. Therefore, the graph of $y^{1}$ restricted to the interval $\left.] t_{1}^{0}, a\right]$ is on the right of the barrier $\Sigma_{1}$.

We consider now the map $w_{2}^{1}:\left[t_{1}^{0}, a\right] \rightarrow \mathbb{R}$ defined as $w_{2}^{1}(t)=\tau_{2}\left(y^{1}(t)\right)-t$, $t \in\left[t_{1}^{0}, a\right]$. By (H1.2) we have

$w_{2}^{1}\left(t_{1}^{0}\right)=\tau_{2}\left(y_{1}\right)-t_{1}^{0}=\tau_{2}\left(y^{0}\left(t_{1}^{0}\right)+I_{1}\left(y^{0}\left(t_{1}^{0}\right)\right)\right)-t_{1}^{0}>\tau_{1}\left(y^{0}\left(t_{1}^{0}\right)\right)-t_{1}^{0}=t_{1}^{0}-t_{1}^{0}=0$

and by (H1.1)

$$
w_{2}^{1}(a)=\tau_{2}\left(y^{1}(a)\right)-a<a-a=0 .
$$

The map $w_{2}^{1}$ is continuous by the continuity of the maps $\tau_{2}$ and $y^{1}$. Therefore, reasoning as in Step 1, we can say that there exists $t_{2}^{1}=\min \{t \in] t_{1}^{0}, a\left[: \omega_{2}^{1}(t)=\right.$ $0\}$.

STEP 3. We proceed iteratively setting for every $j=2, \ldots, m, y_{j}=y^{j-1}\left(t_{j}^{j-1}\right)+$ $I_{j}\left(y^{j-1}\left(t_{j}^{j-1}\right)\right)$ and considering a map $y^{j} \in \mathcal{S}_{\left(t_{j}^{j-1}, y_{j}\right)}$ (cf. (4)), i.e. $y^{j}$ : $\left[t_{j}^{j-1}, a\right] \rightarrow E$ is such that

$$
y^{j}(t)=U\left(t-t_{j}^{j-1}\right) y_{j}+\int_{t_{j}^{j-1}}^{t} U(t-s) g^{j}(s) d s, t \in\left[t_{j}^{j-1}, a\right]
$$

with $g^{j} \in L^{1}\left(\left[t_{j}^{j-1}, a\right] ; E\right), g^{j}(s) \in G\left(s, y^{j}(s)\right)$ for a.e. $s \in\left[t_{j}^{j-1}, a\right]$. We notice that the point $\left(t_{j}^{j-1}, y_{j}\right)$ is on the right or belongs to the barrier $\Sigma_{j}$ and is on the left of the barrier $\Sigma_{j+1}$ (assuming $\Sigma_{m+1}=\{(a, x): x \in E\}$ ). Moreover, the graph of $y^{j}$ restricted to the interval $\left.] t_{j}^{j-1}, a\right]$ is on the right of the barrier $\Sigma_{j}$. Further, the map $y^{j}$ intersects the barrier $\Sigma_{j+1}(j \neq m)$ in $t_{j+1}^{j}=\min \{t \in] t_{j}^{j-1}, a\left[: \omega_{j+1}^{j}(t)=0\right\}$.

STEP 4. We claim that the map $y:[0, a] \rightarrow E$ defined as

$$
y(t)=\left\{\begin{array}{l}
y^{0}(t) \quad t \in\left[0, t_{1}^{0}\right] \\
\left.\left.y^{j}(t) t \in\right] t_{j}^{j-1}, t_{j+1}^{j}\right], j=1, \ldots, m-1 \\
\left.\left.y^{m}(t) t \in\right] t_{m}^{m-1}, a\right]
\end{array}\right.
$$

is a solution of the problem $(I P)$.

To this aim we define the map $g:[0, a] \rightarrow E$ as

$$
g(t)=\left\{\begin{array}{l}
g^{0}(t) \quad t \in\left[0, t_{1}^{0}\right] \\
\left.\left.g^{j}(t) \quad t \in\right] t_{j}^{j-1}, t_{j+1}^{j}\right], j=1, \ldots, m-1 \\
\left.\left.g^{m}(t) t \in\right] t_{m}^{m-1}, a\right] .
\end{array}\right.
$$


It is easy to see that $g \in L^{1}([0, a] ; E)$ and $g(s) \in G(s, y(s))$ for a.e. $s \in[0, a]$. We will prove now that

$$
y(t)=U(t) y_{0}+\sum_{0<t_{j}^{j-1}<t} U\left(t-t_{j}^{j-1}\right) I_{j}\left(y\left(t_{j}^{j-1}\right)\right)+\int_{0}^{t} U(t-s) g(s) d s, t \in[0, a] .
$$

From (6), for $t \in\left[0, t_{1}^{0}\right]$ it is trivial. For $\left.\left.t \in\right] t_{1}^{0}, t_{2}^{1}\right]$ we have

$$
\begin{aligned}
y(t)= & y^{1}(t)=U\left(t-t_{1}^{0}\right)\left[y^{0}\left(t_{1}^{0}\right)+I_{1}\left(y^{0}\left(t_{1}^{0}\right)\right)\right]+\int_{t_{1}^{0}}^{t} U(t-s) g^{1}(s) d s \\
= & U\left(t-t_{1}^{0}\right)\left[U\left(t_{1}^{0}\right) y_{0}+\int_{0}^{t_{1}^{0}} U\left(t_{1}^{0}-s\right) g^{0}(s) d s+I_{1}\left(y^{0}\left(t_{1}^{0}\right)\right)\right] \\
& +\int_{t_{1}^{0}}^{t} U(t-s) g^{1}(s) d s \\
= & U(t) y_{0}+\int_{0}^{t_{1}^{0}} U(t-s) g^{0}(s) d s+U\left(t-t_{1}^{0}\right) I_{1}\left(y^{0}\left(t_{1}^{0}\right)\right) \\
& +\int_{t_{1}^{0}}^{t} U(t-s) g^{1}(s) d s \\
= & U(t) y_{0}+U\left(t-t_{1}^{0}\right) I_{1}\left(y\left(t_{1}^{0}\right)\right)+\int_{0}^{t} U(t-s) g(s) d s .
\end{aligned}
$$

With an iterative method we obtain the claimed result.

As we could see, assumption (H2) was used to obtain (7) and, in consequence, to prove that every trajectory meets each barrier exactly once. Since we consider in $(I P)$ a concrete initial condition, we need slightly less than $(\mathrm{H} 2)$. Below we describe one of possible situations where (7) and analogs for all $j=2, \ldots, m$ are implied without (H2). In what follows, by $\|\cdot\|_{\mathcal{L}(E)}$ we denote a standard norm in the space of linear bounded operators from $E$ to itself and by $B_{X}^{r}(x)$ an open ball in a metric space $X$ centered at $x$ and with radius $r>0$.

Corollary 2.5 Let $E$ be a Banach space, $y_{0} \in E$, and $A: D(A) \subseteq E \rightarrow E$ be a linear operator satisfying (A). Assume that $M_{j}:=\sup _{x \in E}\left\|I_{j}(x)\right\|<\infty$ for every $j=1, \ldots, m$, $G:[0, a] \times E \multimap E$ is a multimap satisfying (G1)-(G3), (G5) and

(G4)' there exists $\alpha \in L_{+}^{1}([0, a])$ such that

$$
\|G(t, x)\| \leq \alpha(t), \text { for a.e. } t \in[0, a] \text { and every } x \in E ;
$$

$\tau_{j}: E \rightarrow \mathbb{R}, j=1, \ldots, m$, are Fréchet differentiable functions satisfying $(\mathrm{H} 1)$ and

(H3) there exists $K>0$ such that $\left\|\tau_{j}^{\prime}(y)\right\| \leq K$, for every $j=1, \ldots, m$ and $y \in$ $\operatorname{cl} B_{E}^{N}\left(y_{0}\right)$,

where $N:=(B+2)\left\|y_{0}\right\|+(B+1) \sum_{j=1}^{m} M_{j}+B\|\alpha\|_{L^{1}}, B:=\sup \left\{\|U(t)\|_{\mathcal{L}(E)}: 0 \leq\right.$ $t \leq a\}<\infty$. Moreover, assume that

(k) $K B \int_{t_{1}}^{t_{2}} \alpha(s) d s+K N\left\|U\left(t_{2}-t_{1}\right)-I\right\|_{\mathcal{L}(E)}<t_{2}-t_{1}$, for each $0 \leq t_{1}<t_{2} \leq a$,

where $\alpha$ and $K$ are from $(\mathrm{G} 4)$ ' and (H3), respectively.

Then the problem (I P) admits at least one mild solution on $[0, a]$. 
Proof As in the proof of Theorem 2.4, let $y^{0} \in \mathcal{S}_{\left(0, y_{0}\right)}$. Up to the first impulse time $t_{1}^{0}$ we have

$$
\left\|y^{0}(t)-y_{0}\right\| \leq\|U(t)-I\|_{\mathcal{L}(E)}\left\|y_{0}\right\|+B \int_{0}^{t} \alpha(s) d s \leq(B+1)\left\|y_{0}\right\|+B\|\alpha\|_{L^{1}} \leq N,
$$

for every $t \in[0, a]$. So, denoted $y_{1}=y^{0}\left(t_{1}^{0}\right)+I_{1}\left(y^{0}\left(t_{1}^{0}\right)\right)$, we get

$$
\left\|y_{1}-y_{0}\right\| \leq M_{1}+(B+1)\left\|y_{0}\right\|+B\|\alpha\|_{L^{1}} \leq N
$$

and

$$
\left\|y_{1}\right\| \leq M_{1}+(B+2)\left\|y_{0}\right\|+B\|\alpha\|_{L^{1}} \leq N .
$$

Now we consider a map $y^{1} \in \mathcal{S}_{\left(t_{1}^{0}, y_{1}\right)}$,

$$
y^{1}(t)=U\left(t-t_{1}^{0}\right) y_{1}+\int_{t_{1}^{0}}^{t} U(t-s) g^{1}(s) d s, t \in\left[t_{1}^{0}, a\right]
$$

with $g^{1} \in L^{1}\left(\left[t_{1}^{0}, a\right] ; E\right), g^{1}(s) \in G\left(s, y^{1}(s)\right)$ for a.e. $s \in\left[t_{1}^{0}, a\right]$. Defining the function

$$
g_{1}(t)=\left\{\begin{array}{l}
g^{0}(t) t \in\left[0, t_{1}^{0}\right] \\
\left.\left.g^{1}(t) t \in\right] t_{1}^{0}, a\right]
\end{array}\right.
$$

by means of analogous calculations as in (9) we get

$$
y^{1}(t)=U(t) y_{0}+U\left(t-t_{1}^{0}\right) I_{1}\left(y^{0}\left(t_{1}^{0}\right)\right)+\int_{0}^{t} U(t-s) g_{1}(s) d s, t \in\left[t_{1}^{0}, a\right] .
$$

Moreover, for $\left.t \in] t_{1}^{0}, a\right]$ we have

$$
\begin{aligned}
\left\|y^{1}(t)-y_{0}\right\| & \leq\left\|U(t) y_{0}-y_{0}+U\left(t-t_{1}^{0}\right) I_{1}\left(y^{1}\left(t_{1}^{0}\right)\right)+\int_{0}^{t} U(t-s) g_{1}(s) d s\right\| \\
& \leq(B+1)\left\|y_{0}\right\|+B M_{1}+B\|\alpha\|_{L^{1}} \leq N .
\end{aligned}
$$

Hence, for every $\left.t \in] t_{1}^{0}, a\right]$, thanks to (13) and (10) we can use (H3); so, by (11), (12), and $(\mathrm{k})$ we obtain

$$
\begin{aligned}
& \tau_{1}\left(y^{1}(t)\right)-t-\left(\tau_{1}\left(y_{1}\right)-t_{1}^{0}\right) \leq K\left\|y^{1}(t)-y_{1}\right\|-\left(t-t_{1}^{0}\right) \\
& \quad \leq K\left\|\int_{t_{1}^{0}}^{t} U(t-s) g^{1}(s) d s\right\|-\left(t-t_{1}^{0}\right)+K\left\|U\left(t-t_{1}^{0}\right)-I\right\|_{\mathcal{L}(E)}\left\|y_{1}\right\| \\
& \quad \leq \quad K B \int_{t_{1}^{0}}^{t} \alpha(s) d s-\left(t-t_{1}^{0}\right)+K N\left\|U\left(t-t_{1}^{0}\right)-I\right\|_{\mathcal{L}(E)}<0 .
\end{aligned}
$$

Hence, as in the proof of Theorem 2.4, we know that the graph of $y^{1}$ is on the right of $\Sigma_{1}$, and the next impulse time $t_{2}^{1}$ is the time of a meeting point with $\Sigma_{2}$.

Also for $y_{2}=y^{1}\left(t_{2}^{1}\right)+I_{2}\left(y^{1}\left(t_{2}^{1}\right)\right)$ we obtain

$$
\left\|y_{2}-y_{0}\right\| \leq M_{2}+(B+1)\left\|y_{0}\right\|+B M_{1}+B\|\alpha\|_{L^{1}} \leq N
$$

and

$$
\left\|y_{2}\right\| \leq M_{2}+(B+2)\left\|y_{0}\right\|+B M_{1}+B\|\alpha\|_{L^{1}} \leq N,
$$

so the map $y^{2} \in \mathcal{S}_{\left(t_{2}^{1}, y_{2}\right)}$ satisfies the following inequality

$$
\left.\left.\tau_{2}\left(y^{2}(t)\right)-t-\tau_{2}\left(y_{2}\right)+t_{2}^{1}<0 \quad \text { for every } t \in\right] t_{2}^{1}, a\right] .
$$

Thus (5) is satisfied by $y^{2}$. Then, proceeding iteratively, we can build a function $y$ as in (8). Like in the proof of Theorem 2.4, this map is a solution to problem (IP). 


\section{Existence Results via Equi-Lyapunov Pairs}

Let $M$ be a nonempty set in a Banach space $E, G:[0, a] \times M \multimap E$ be a given multimap. Consider the non-autonomous semilinear differential inclusion

$$
y^{\prime}(t) \in A y(t)+G(t, y(t)) \text {, for a.e. } t \in[0, a]
$$

and suppose that there exists at least one global mild solution of (14). Notice that the existence of such a solution is guaranteed for example under assumptions (G1)-(G5) (see Remark 2.2).

In order to study a characterization of equi-Lyapunov pairs of (14) (see Definition 1.3), we need at first to consider an autonomous case.

\subsection{Sufficient Conditions for Equi-Lyapunov Pairs: Autonomous Case}

In this subsection we consider the following autonomous semilinear differential inclusion

$$
y^{\prime}(t) \in A y(t)+G(y(t)), \text { for a.e. } t \in[0, a],
$$

where $G: M \multimap E$ and we obtain sufficient conditions to have equi-Lyapunov pairs for (15) . Precisely we have the following result.

Theorem 3.1 Let $E$ be a separable Banach space, $A: D(A) \subseteq E \rightarrow E$ be a linear operator satisfying (A), $G: E \multimap E$ be a nonempty closed bounded valued multimap, $V: E \rightarrow \mathbb{R}$ be a lower semicontinuous function, and $p: E \rightarrow \mathbb{R}$ be a function. If

(i) for every bounded set $\Omega \subset E$ there exists a constant $L_{\Omega}>0$ such that

$$
G(x) \subset G(y)+L_{\Omega}\|x-y\| B_{E}^{1}(0), \text { for every } x, y \in \Omega
$$

and

$$
|p(x)-p(y)| \leq L_{\Omega}\|x-y\|, \text { for every } x, y \in \Omega
$$

(ii) for every $\xi \in E$ and every $f \in G(\xi)_{L^{1}}$ the next inequality holds

$$
\underline{D}^{A} V(\xi)(f)+p(\xi) \leq 0
$$

then $(V, p)$ is an equi-Lyapunov pair for inclusion (15).

Proof Let $X=E \times \mathbb{R}$ be the separable Banach space endowed with the norm $\|(\xi, s)\|_{X}=$ $\max \{\|\xi\|,|s|\}$, for every $(\xi, s) \in X$, and let $F: X \multimap X$ be the multimap defined as

$$
F(\xi, s)=G(\xi) \times\{-p(\xi)\},(\xi, s) \in X .
$$

We claim that $F$ is Lipschitz on bounded sets, i.e. for every $(\xi, r) \in X$ and for every $R>0$ there exists a constant $L_{R}>0$ such that

$$
F(x, s) \subset F(y, t)+L_{R}\|(x, s)-(y, t)\|_{X} B_{X}^{1}(0),
$$

for every $(x, s),(y, t) \in B_{E}^{R}(\xi) \times B_{\mathbb{R}}^{R}(r)$. Indeed, let $(\xi, r) \in X, R>0$ and $(x, s),(y, t) \in$ $B_{E}^{R}(\xi) \times B_{\mathbb{R}}^{R}(r)$. From assumption (i) there exists $L_{R}>0$ such that

$$
\begin{aligned}
F(x, s)=G(x) \times\{-p(x)\} & \subset G(y) \times\{-p(y)\}+L_{R}\|x-y\|\left(B_{E}^{1}(0) \times B_{\mathbb{R}}^{1}(0)\right) \\
& \subset F(y, t)+L_{R}\|(x, s)-(y, t)\|_{X} B_{X}^{1}(0) .
\end{aligned}
$$

Let $\mathscr{A}: D(\mathscr{A}) \subseteq X \rightarrow X$ be the linear operator defined as $D(\mathscr{A})=D(A) \times \mathbb{R}, \mathscr{A}=$ $(A, 0)$. It is known that $\mathscr{A}$ is the infinitesimal generator of the $C_{0}$-semigroup $\{\mathscr{U}(t)\}_{t \geq 0}=$ 
$\left\{\left(U(t), 1_{\mathbb{R}}\right)\right\}_{t \geq 0}$. Let $K=$ epi $V$, we will prove now that for every $(\xi, r) \in K$ we have (see (1))

$$
F(\xi, r)_{L^{1}} \subset \mathscr{F}_{K}^{\mathscr{A}}(\xi, r),
$$

where $\mathscr{F}_{K}^{\mathscr{A}}$ is the class of all $\mathscr{A}$-tangent functions to $K$ at $(\xi, r)$ introduced in the next Appendix.

Let $(\xi, r) \in K$ and $f \in F(\xi, r)_{L^{1}}$, i.e. there exists $g \in L_{l o c}^{1}\left(\mathbb{R}^{+} ; E\right)$ with $g(s) \in G(\xi)$ for a.e. $s \in \mathbb{R}^{+}$, such that

$$
f(s)=(g(s),-p(\xi)), s \in \mathbb{R}^{+} .
$$

By (ii) we have that (see Definition 1.4)

$$
\liminf _{\substack{h \downarrow 0 \\ w \rightarrow 0_{E}}} \frac{1}{h}\left[V\left(U(h) \xi+\int_{0}^{h} U(h-s) g(s) d s+h w\right)-V(\xi)\right] \leq-p(\xi) .
$$

That is, there exist two sequences $\left\{h_{n}\right\}_{n} \subset \mathbb{R}^{+}, h_{n} \downarrow 0$ and $\left\{w_{n}\right\}_{n} \subset E, w_{n} \rightarrow 0_{E}$ such that for every $n \in \mathbb{N}$

$$
V\left(U\left(h_{n}\right) \xi+\int_{0}^{h_{n}} U\left(h_{n}-s\right) g(s) d s+h_{n} w_{n}\right) \leq V(\xi)+h_{n}\left(-p(\xi)+\frac{1}{n}\right) .
$$

Hence, for every $n \in \mathbb{N}$

$$
\left(U\left(h_{n}\right) \xi+\int_{0}^{h_{n}} U\left(h_{n}-s\right) g(s) d s+h_{n} w_{n}, V(\xi)+h_{n}\left(-p(\xi)+\frac{1}{n}\right)\right) \in K .
$$

By the definition of the $C_{0}$-semigroup $\{\mathscr{U}(t)\}_{t \geq 0}$ and by (17) this implies that

$$
\mathscr{U}\left(h_{n}\right)(\xi, V(\xi))+\int_{0}^{h_{n}} \mathscr{U}\left(h_{n}-s\right) f(s) d s+h_{n}\left(w_{n}, \frac{1}{n}\right) \in K .
$$

Therefore, by Remark A.2, we have proven that for every $\xi \in E, f(\cdot)=(g(\cdot),-p(\xi)) \in$ $\mathscr{F}_{K}^{\mathscr{A}}(\xi, V(\xi))$.

We can estimate the right hand side of $(18)$ by $\rho+h_{n}\left(-p(\xi)+\frac{1}{n}\right)$ for every $\rho \geq V(\xi)$, which implies

$$
\mathscr{F}_{K}^{\mathscr{A}}(\xi, V(\xi)) \subset \bigcap_{\rho \geq V(\xi)} \mathscr{F}_{K}^{\mathscr{A}}(\xi, \rho) \subset \mathscr{F}_{K}^{\mathscr{A}}(\xi, r),
$$

therefore (16) holds. By Theorem A.3 with the uniqueness function defined as $\omega_{R}(s)=$ $L_{R} s, s \in \mathbb{R}_{0}^{+}$, we have that $K$ is invariant with respect to $\mathscr{A}+F$, i.e. for every $(\tau, \xi, r) \in$ $[0, a[\times K$ and every mild solution $w:[\tau, a] \rightarrow X, w=(y, z)$ of

$$
\left\{\begin{array}{l}
y^{\prime}(t) \in A y(t)+G(y(t)), t \in[\tau, a] \\
z^{\prime}(t)=-p(y(t)), t \in[\tau, a] \\
y(\tau)=\xi \\
z(\tau)=r
\end{array}\right.
$$

we have $w(t)=(y(t), z(t)) \in K$ for every $t \in[\tau, a]$. Hence $V(y(t)) \leq z(t)$ for every $t \in[\tau, a]$. From

$$
z(t)=r-\int_{\tau}^{t} p(y(s)) d s, \text { for all } t \in[\tau, a]
$$

we get for every mild solution $y$ of (15) such that $y(\tau)=\xi$

$$
V(y(t))+\int_{\tau}^{t} p(y(s)) d s \leq r, \text { for all } t \in[\tau, a] \text { and for all } r \geq V(\xi),
$$


in particular, with $r=V(\xi)$ we obtain the claimed result.

We can prove an analogous result with $p$ a lower semicontinuous map.

Theorem 3.2 Let E be a separable Banach space, A be a linear operator satisfying (A), $G: E \multimap E$ be a nonempty closed bounded valued multimap, and $V, p: E \rightarrow \mathbb{R}$ be lower semicontinuous functions. If

(i)' for every bounded set $\Omega \subset E$ there exists a constant $L_{\Omega}>0$ such that

$$
G(x) \subset G(y)+L_{\Omega}\|x-y\| B_{E}^{1}(0), \text { for every } x, y \in \Omega ;
$$

(ii) for every $\xi \in E$ and every $f \in G(\xi)_{L^{1}}$ the next inequality holds

$$
\underline{D}^{A} V(\xi)(f)+p(\xi) \leq 0 ;
$$

(iii) there exist $C>0, r \geq 1$ such that

$$
p(x) \geq-C\left(1+\|x\|^{r}\right), \text { for every } x \in E ;
$$

then $(V, p)$ is an equi-Lyapunov pair for inclusion (15).

Proof By Lemma A.1 there exists a sequence of functions $\left\{p_{n}\right\}_{n}, p_{n}: E \rightarrow \mathbb{R}$ such that $p_{n}$ is Lipschitz continuous on bounded sets of $E$ for every $n \in \mathbb{N}$ and $p_{n} \uparrow p$ pointwise in $E$ as $n$ goes to infinity. By condition (ii) we have that for every $\xi \in E$ and every $f \in G(\xi)_{L^{1}}$

$$
\underline{D}^{A} V(\xi)(f)+p_{n}(\xi) \leq \underline{D}^{A} V(\xi)(f)+p(\xi) \leq 0 \text {, for every } n \in \mathbb{N} .
$$

By Theorem 3.1 we have that $\left(V, p_{n}\right)$ is an equi-Lyapunov pair for (15). From Definition 1.3 it follows that for every $(\tau, \xi) \in[0, a[\times E$ and every mild solution $y:[\tau, a] \rightarrow E$ to the multivalued semilinear Cauchy problem $(P)_{(\tau, \xi)}$

$$
V(y(t))+\int_{\tau}^{t} p_{n}(y(s)) d s \leq V(\xi), t \in[\tau, a] .
$$

Then

$$
V(y(t))+\int_{\tau}^{t} p(y(s)) d s=\limsup _{n \rightarrow+\infty}\left(V(y(t))+\int_{\tau}^{t} p_{n}(y(s)) d s\right) \leq V(\xi),
$$

for $t \in[\tau, a]$, obtaining that $(V, p)$ is an equi-Lyapunov pair for (15).

\subsection{Sufficient Conditions for Equi-Lyapunov Pairs: Non-autonomous Case}

In this section, by using the results obtained in Section 3.1, we provide analogous results in the non-autonomous case (14).

Theorem 3.3 Let $E$ be a separable Banach space, A be a linear operator satisfying (A), $G:[0, a] \times E \multimap E$ be a nonempty closed bounded valued multimap, $V: E \rightarrow \mathbb{R}$ be $a$ lower semicontinuous function, and $p: E \rightarrow \mathbb{R}$ be a function. If

(j) for every bounded set $\Omega \subset[0, a] \times E$ there exists a constant $L_{\Omega}>0$ such that

$$
G(t, x) \subset G(s, y)+L_{\Omega} \max \{|t-s|,\|x-y\|\} B_{E}^{1}(0), \text { for every }(t, x),(s, y) \in \Omega
$$

and for every bounded set $\Omega^{\prime} \subset E$ there exists a constant $L_{\Omega^{\prime}}>0$ such that

$$
|p(x)-p(y)| \leq L_{\Omega^{\prime}}\|x-y\| \text {, for every } x, y \in \Omega^{\prime} ;
$$


(ji) for every $(\tau, \xi) \in[0, a] \times E$ and every $f \in G(\tau, \xi)_{L^{1}}$ the next inequality holds

$$
\underline{D}^{A} V(\xi)(f)+p(\xi) \leq 0 ;
$$

then $(V, p)$ is an equi-Lyapunov pair for inclusion (14).

Proof Let $Y=\mathbb{R} \times E$ be the separable Banach space endowed with the norm $\|z\|=$ $\|(t, x)\|_{Y}=\max \{|t|,\|x\|\}$, for every $(t, x) \in Y, \mathcal{A}: D(\mathcal{A}) \subseteq Y \rightarrow Y$ be the linear operator defined as $D(\mathcal{A})=\mathbb{R} \times D(A), \mathcal{A}=(0, A)$, that is, $\mathcal{A}(t, x)=(0, A x)$. It is known that $\mathcal{A}$ is the infinitesimal generator of the $C_{0}$-semigroup $\{\mathcal{U}(s)\}_{s \geq 0}=\left\{\left(1_{\mathbb{R}}, U(s)\right)\right\}_{s \geq 0}$, $\mathcal{U}(s) z=(t, U(s) x)$ for every $s \geq 0$, where $z=(t, x)$ and $\{U(s)\}_{s \geq 0}$ is the $C_{0}$-semigroup from (A). Let $\mathcal{G}: Y \multimap Y$ be the multimap defined as

$$
\mathcal{G}(t, x)=\left\{\begin{array}{l}
\{1\} \times G(t, x) \text { for } t \in[0, a], \\
\{1\} \times G(0, x) \text { for } t<0, \\
\{1\} \times G(a, x) \text { for } t>a
\end{array}\right.
$$

It is not hard to check that $\mathcal{G}$ satisfies assumption (i) from Theorem 3.1. Indeed, denote by $\pi_{[0, a]}$ a metric projection of $\mathbb{R}$ onto $[0, a]$, and by $\pi_{[0, a] \times E}: Y \rightarrow[0, a] \times E$ the map $\pi_{[0, a] \times E}(t, x):=\left(\pi_{[0, a]}(t), x\right)$. Given $\Omega \subset Y$ an arbitrary bounded set, the set $\tilde{\Omega}:=$ $\pi_{[0, a] \times E}(\Omega)$ is a bounded subset of $[0, a] \times E$. Let $L_{\Omega}:=L_{\tilde{\Omega}}$ be the Lipschitz constant from (j). Then, for any $(s, y),(\tau, \xi) \in \Omega$,

$$
\begin{aligned}
\mathcal{G}(s, y) & =\{1\} \times G\left(\pi_{[0, a]}(s), y\right) \\
& \subset\{1\} \times G\left(\pi_{[0, a]}(\tau), \xi\right)+\{0\} \times L_{\Omega} \max \left\{\left|\pi_{[0, a]}(s)-\pi_{[0, a]}(\tau)\right|,\|y-\xi\|\right\} B_{E}^{1}(0) \\
& \subset \mathcal{G}(\tau, \xi)+L_{\Omega} \max \left\{\left|\pi_{[0, a]}(s)-\pi_{[0, a]}(\tau)\right|,\|y-\xi\|\right\} B_{\mathbb{R} \times E}^{1}(0) \\
& \subset \mathcal{G}(\tau, \xi)+L_{\Omega} \max \{|s-\tau|,\|y-\xi\|\} B_{\mathbb{R} \times E}^{1}(0) .
\end{aligned}
$$

Hence letting $(\tau, \xi) \in[0, a[\times E$ be fixed and $z:[0, a-\tau] \rightarrow Y$ be defined as $z(s)=$ $(t(s), y(s)), s \in[0, a-\tau]$ we can rewrite the problem $(P)_{(\tau, \xi)}$ as the following autonomous problem in the separable Banach space $Y$ :

$$
(P)_{(0, \zeta)}\left\{\begin{array}{l}
z^{\prime}(s) \in \mathcal{A} z(s)+\mathcal{G}(z(s)), \text { a.e. } s \in[0, a-\tau] \\
z(0)=\zeta=(\tau, \xi) .
\end{array}\right.
$$

Indeed, the above problem is equivalent to

$$
\left\{\begin{array}{l}
t^{\prime}(s)=1 \\
y^{\prime}(s) \in A y(s)+G(t(s), y(s)), \text { a.e. } s \in[0, a-\tau] \\
t(0)=\tau \\
y(0)=\xi
\end{array}\right.
$$

that is

$$
\left\{\begin{array}{l}
t(s)=s+\tau \\
y^{\prime}(s) \in A y(s)+G(s+\tau, y(s)), \text { a.e. } s \in[0, a-\tau] \\
y(0)=\xi .
\end{array}\right.
$$

and we can obtain $(P)_{(\tau, \xi)}$ setting $t=s+\tau$ and $x:[\tau, a] \rightarrow E$ so defined $x(t)=y(t-\tau)$. To see this we notice that a mild solution of $(P)_{(0, \zeta)}$ is given by

$$
\begin{aligned}
z(s) & =(t(s), y(s))=\mathcal{U}(s) \zeta+\int_{0}^{s} \mathcal{U}(s-\sigma)(1, g(\sigma)) d \sigma \\
& =\left(s+\tau, U(s) \xi+\int_{0}^{s} U(s-\sigma) g(\sigma) d \sigma\right), s \in[0, a-\tau]
\end{aligned}
$$


where $g \in L^{1}([0, a] ; E), g(s) \in G(t(s), y(s))$ for a.e. $s \in[0, a-\tau]$. Since $t(s)=s+\tau$, we have

$$
x(t)=y(t-\tau)=U(t-\tau) \xi+\int_{\tau}^{t} U(t-r) g(r-\tau) d r, t \in[\tau, a]
$$

where $g(r-\tau) \in G(t(r-\tau), y(r-\tau))=G(r, x(r))$. Hence, $x$ is a mild solution of $(P)_{(\tau, \xi)}$. In consequence, any solution $z(\cdot)$ to $(P)_{(0, \zeta)}$ can be written as

$$
z(s)=(t(s), y(s))=(t(s), y(t(s)-\tau))=(t(s), x(t(s))),
$$

where $x:[\tau, a] \rightarrow E$ is a mild solution of $(P)_{(\tau, \xi)}$.

Let $f \in \mathcal{G}(\zeta)_{L^{1}}$, i.e., $f=(1, \widetilde{f})$, with $\widetilde{f} \in G(\tau, \xi)_{L^{1}}$. Setting $\omega=(t, w) \in[0, a] \times E$, we have that

$$
\begin{aligned}
& \mathcal{U}(h) \zeta+\int_{0}^{h} \mathcal{U}(h-s) f(s) d s+h \omega \\
& =(\tau, U(h) \xi)+\left(h, \int_{0}^{h} U(h-s) \tilde{f}(s) d s\right)+h(t, w) \\
& =\left(\tau+h+h t, U(h) \xi+\int_{0}^{h} U(h-s) \tilde{f}(s) d s+h w\right), \text { for every } h \in \mathbb{R}^{+} .
\end{aligned}
$$

Therefore, defining $\mathcal{V}: Y \rightarrow \mathbb{R}$ as $\mathcal{V}(z) \equiv \mathcal{V}(t, x)=V(x)$, for every $z=(t, x) \in Y$, we have

$$
\begin{aligned}
& \mathcal{V}\left(\mathcal{U}(h)(\zeta)+\int_{0}^{h} \mathcal{U}(h-s) f(s) d s+h \omega\right) \\
= & V\left(U(h) \xi+\int_{0}^{h} U(h-s) \tilde{f}(s) d s+h w\right)
\end{aligned}
$$

for every $h \in \mathbb{R}^{+}$. Thus, defining $\mathcal{P}: Y \rightarrow \mathbb{R}$ as $\mathcal{P}(z) \equiv \mathcal{P}(t, x)=p(x)$, for every $z=(t, x) \in Y$, by $(\mathrm{jj})$ it follows that

$$
\underline{D}^{\mathcal{A}} \mathcal{V}(\zeta)(f)+\mathcal{P}(\zeta) \leq 0
$$

Hence, by the arbitrariness of $\zeta$ and $f$, we can apply Theorem 3.1 and so we obtain that $(\mathcal{V}, \mathcal{P})$ is an equi-Lyapunov pair for the inclusion

$$
z^{\prime}(s) \in \mathcal{A} z(s)+\mathcal{G}(z(s)), \text { a.e. } s \in[0, a-\tau],
$$

i.e. for any $\zeta=(\tau, \xi) \in Y$ and for any mild solution $z:[0, a-\tau] \rightarrow Y$ of $(P)_{(0, \zeta)}$ we have

$$
\mathcal{V}(z(s))+\int_{0}^{s} \mathcal{P}(z(r)) d r \leq \mathcal{V}(\zeta), s \in[0, a-\tau] .
$$

Therefore, recalling that $z(s)=(t(s), x(t(s)))$ for every $s \in[0, a-\tau]$, where $x:[\tau, a] \rightarrow$ $E$ is a mild solution of $(P)_{(\tau, \xi)}$, it follows that

$$
V(x(s+\tau))+\int_{0}^{s} p(x(r+\tau)) d r \leq V(\xi), s \in[0, a-\tau] .
$$

Hence, setting $r+\tau=\sigma$ we obtain

$$
V(x(s+\tau))+\int_{\tau}^{s+\tau} p(x(\sigma)) d \sigma \leq V(\xi), s \in[0, a-\tau] .
$$


Finally, recalling that $t=s+\tau$ we have

$$
V(x(t))+\int_{\tau}^{t} p(x(\sigma)) d \sigma \leq V(\xi), t \in[0, a],
$$

concluding the proof.

With similar arguments we can prove a result analogous to the previous one, but involving a map $p$ as in Theorem 3.2.

Theorem 3.4 Let $E$ be a separable Banach space, A be a linear operator satisfying (A), $G:[0, a] \times E \multimap E$ be a nonempty closed bounded valued multimap, and $V, p: E \rightarrow \mathbb{R}$ be lower semicontinuous functions. If

(j) for every bounded set $\Omega \subset[0, a] \times E$ there exists a constant $L_{\Omega}>0$ such that

$$
G(t, x) \subset G(s, y)+L_{\Omega} \max \{|t-s|,\|x-y\|\} B_{E}^{1}(0), \text { for every }(t, x),(s, y) \in \Omega \text {; }
$$

(jj) for every $(\tau, \xi) \in[0, a] \times E$ and for every $f \in G(\tau, \xi)_{L^{1}}$ the next inequality holds

$$
\underline{D}^{A} V(\xi)(f)+p(\xi) \leq 0 ;
$$

(iii) there exist $C>0, r \geq 1$ such that

$$
p(x) \geq-C\left(1+\|x\|^{r}\right), \text { for every } x \in E ;
$$

then $(V, p)$ is an equi-Lyapunov pair for inclusion (14).

\subsection{Existence of Impulsive Mild Solutions}

In this Section we give existence results for problem $(I P)$ via equi-Lyapunov pairs.

Firstly, observe that it is possible to provide results analogous to Theorems 3.1-3.4 obtaining the existence of an equi-Lyapunov pair for problem $(P)_{(\tau, \xi)}$ (see Definition 1.5). We state the analog to Theorem 3.4, since we will need it in the sequel.

Corollary 3.5 Let $E$ be a separable Banach space, $A: D(A) \subseteq E \rightarrow E$ be a linear operator satisfying $(A), G:[0, a] \times E \multimap E$ be a nonempty closed bounded valued multimap satisfying (j)', $V: E \rightarrow \mathbb{R}$ be a lower semicontinuous function and $(\tau, \xi) \in[0, a[\times E$. If there exists a lower semicontinuous function $\left.p_{(\tau, \xi)}: E \rightarrow\right] 0,+\infty[$ such that

(jj)' for every $(\theta, \eta) \in[0, a] \times E$ and $f \in G(\theta, \eta)_{L^{1}}$ the next inequality holds

$$
\underline{D}^{A} V(\eta)(f)+p_{(\tau, \xi)}(\eta) \leq 0 ;
$$

(iii) there exist $C>0, r \geq 1$ such that

$$
p_{(\tau, \xi)}(x) \geq-C\left(1+\|x\|^{r}\right), \text { for every } x \in E ;
$$

then, $\left(V, p_{(\tau, \xi)}\right)$ is an equi-Lyapunov pair for problem $(P)_{(\tau, \xi)}$.

The proof of the above corollary is the same as the one of Theorem 3.4, since the fact that $(\tau, \xi)$ is fixed in the hypotheses leads us to achieve a weaker thesis in the corollary with respect to the one of the corresponding theorem, i.e. an equi-Lyapunov pair for the Cauchy problem $(P)_{(\tau, \xi)}$ (see Definition 1.5) instead of for the inclusion (see Definition 1.3).

Now, we can provide the results on the existence of mild solutions for problem $(I P)$ by using equi-Lyapunov pairs in the weak sense of Definition 1.5. 
Theorem 3.6 Let $E$ be a separable Banach space, $A: D(A) \subseteq E \rightarrow E$ be a linear operator satisfying (A). Assume that $G:[0, a] \times E \multimap E$ is a multimap satisfying (G1), (G4) and hypothesis (j)' of Theorem 3.4, and that $\tau_{j}: E \rightarrow \mathbb{R}, j=1, \ldots, m$, are functions satisfying (H1). Suppose that

(H2)' for every $j=1, \ldots, m$, and $(\tau, \xi) \in[0, a[\times E$, there exists a lower semicontinuous function $\left.p_{j,(\tau, \xi)}: E \rightarrow\right] 0,+\infty\left[\right.$ such that the couple $\left(\tau_{j}, p_{j,(\tau, \xi)}-1\right)$ is an equiLyapunov pair for problem $(P)_{(\tau, \xi)}$.

Then the problem (IP) admits at least one mild solution on the whole interval $[0, a]$.

Proof Let us note that condition (H2)' means that

for every $j=1, \ldots, m,(\tau, \xi) \in\left[0, a\left[\times E\right.\right.$ and every $y_{(\tau, \xi)} \in \mathcal{S}_{(\tau, \xi)}$ (see (4)) the next inequality holds:

$$
\tau_{j}\left(y_{(\tau, \xi)}(t)\right)+\int_{\tau}^{t} p_{j,(\tau, \xi)}\left(y_{(\tau, \xi)}(s)\right) d s-(t-\tau) \leq \tau_{j}(\xi), \text { for every } t \in[\tau, a] .
$$

Hence, since $p_{j,(\tau, \xi)}$ takes positive values, we deduce that:

for every $j=1, \ldots, m,(\tau, \xi) \in\left[0, a\left[\times E\right.\right.$, and every $y_{(\tau, \xi)} \in \mathcal{S}_{(\tau, \xi)}$ it holds that

$$
\left.\left.\tau_{j}\left(y_{(\tau, \xi)}(t)\right)-t<\tau_{j}(\xi)-\tau, \text { for every } t \in\right] \tau, a\right],
$$

which is property $(\mathrm{H} 2)$.

Now, by the hypothesis (j)' of Theorem 3.4 the multimap $G$ is trivially upper semicontinuous with respect to the second variable, implying assumption (G3), and measurable with respect to the first. Hence by the separability of the space $E$ and the Kuratowski-RyllNardzewski Theorem (see, e.g. [22, Ch. 2, Theorem 2.1]) the multimap $G$ satisfies also assumption (G2) (see [23, Theorem 1.3.1]). Moreover, the hypothesis (j)' implies that $G$ satisfies (G5). Indeed, let $D \subset E$ be a bounded set, $\varepsilon>0, S=\left\{x_{1}, \ldots, x_{q_{\delta(\varepsilon)}}\right\}$ a finite $\delta(\varepsilon)$-net of $D$ with

$$
\delta(\varepsilon):=\chi(D)+\varepsilon,
$$

so that

$$
D \subset \bigcup_{i=1}^{q_{\delta(\varepsilon)}} B\left(x_{i}, \delta(\varepsilon)\right)
$$

Let $t \in[0, a]$, and $z \in G(t, D)$ be fixed, hence there exists $x \in D$ such that $z \in G(t, x)$. By (j)' with $t=s$ and $y=x_{j}, j=1, \ldots, q_{\delta(\varepsilon)}$ it holds

$$
z \in G(t, x) \subset G\left(t, x_{j}\right)+L_{D} \delta(\varepsilon) B_{E}^{1}(0) \subset \bigcup_{j=1}^{q_{\delta(\varepsilon)}} G\left(t, x_{j}\right)+L_{D} \delta(\varepsilon) B_{E}^{1}(0) .
$$

Put $K=\bigcup_{j=1}^{q \delta(\varepsilon)} G\left(t, x_{j}\right)$, this set is compact and the family of balls

$$
\left\{B_{E}^{L_{D} \delta(\varepsilon)}(y): y \in K\right\}
$$

is an open cover of $K$. So we can extract a finite sub-cover, i.e. there exist $y_{1}^{\varepsilon}, \ldots, y_{m(\varepsilon)}^{\varepsilon} \in K$ such that

$$
K \subset \bigcup_{i=1}^{m(\varepsilon)} B_{E}^{L_{D} \delta(\varepsilon)}\left(y_{i}^{\varepsilon}\right)
$$


Hence by (22) and (23) we have

$$
G(t, D) \subset \bigcup_{i=1}^{m(\varepsilon)} B_{E}^{L_{D} \delta(\varepsilon)}\left(y_{i}^{\varepsilon}\right)+L_{D} \delta(\varepsilon) B_{E}^{1}(0) \subset \bigcup_{i=1}^{m(\varepsilon)} B_{E}^{2 L_{D} \delta(\varepsilon)}\left(y_{i}^{\varepsilon}\right) .
$$

Therefore the set $\left\{y_{i}^{\varepsilon}: i=1, \ldots, n\right\}$ is a finite $2 L_{D} \delta(\varepsilon)$-net of $G(t, D)$, obtaining, by the definition of $\delta(\varepsilon)$ (see (21)) that

$$
\chi(G(t, D)) \leq 2 L_{D}(\chi(D)+\varepsilon) .
$$

By the arbitrariness of $\varepsilon>0$ we achieve condition (G5) with $\beta(t)=2 L_{D}$ for every $t \in$ $[0, a]$.

So, we can apply Theorem 2.4 and obtain the claimed result.

We finish with the result where the analytic sufficient condition for assumption (H2)' is provided.

Theorem 3.7 Let $E$ be a separable Banach space, $A: D(A) \subseteq E \rightarrow E$ be a linear operator satisfying (A). Assume that $G:[0, a] \times E \multimap E$ is a multimap satisfying (G1), (G4) and hypothesis (j)' of Theorem 3.4, and that $\tau_{j}: E \rightarrow \mathbb{R}, j=1, \ldots, m$, are functions satisfying (H1). Suppose that

(H2)' for every $j=1, \ldots, m,(\tau, \xi) \in[0, a[\times E$, there exists a lower semicontinuous function $\left.p_{j,(\tau, \xi)}: E \rightarrow\right] 0,+\infty[$ such that for every $(\theta, \eta) \in[0, a] \times E$ and every $f \in G(\theta, \eta)_{L^{1}}$ the next inequality holds

$$
\underline{D}^{A} \tau_{j}(\eta)(f)+p_{j,(\tau, \xi)}(\eta)-1 \leq 0 .
$$

Then the problem (I P) admits at least one mild solution on the whole interval $[0, a]$.

Proof For every $j=1, \ldots, m$ and $(\tau, \xi) \in[0, a[\times E$, by (j)' and (H2)" we have that all hypotheses of Corollary 3.5 are satisfied taking $V=\tau_{j}$ and $p_{(\tau, \xi)}=p_{j,(\tau, \xi)}-1$. So we can say that the couple $\left(\tau_{j}, p_{j,(\tau, \xi)}-1\right)$ is an equi-Lyapunov pair for problem $(P)_{(\tau, \xi)}$, i.e. (H2)' is satisfied. Hence, by Theorem 3.6 we obtain the claimed result.

\section{Concluding Remarks}

The research presented in the preceding sections leads us to several open problems and motivates to a further study. At first, the case where the multivalued perturbation $G$ (see problem $(I P))$ is only measurable with respect to the first variable and sufficiently regular (e.g., lipschitzian) with respect to the second one should be examined. Of course, different method of proof (other than in Theorem 3.3) has to be found.

The second direction of research focuses on non-compact valued perturbations and, instead, compact semigroups or compact evolution systems. We deeply believe it is possible to obtain important analogous results in this case and we take it as a subject of the future study. This development will open an opportunity to examine properties of solutions of new kinds of evolution PDEs with state-dependent impulses.

As the third open problem we can mention the question on topological properties of the mild solution set to problem $(I P)$. Especially, a compactness, contractibility or $R_{\delta}$-property would be welcome under different types of assumptions.

We hope our paper and the above remarks will be considered interesting and inspiring. 
Acknowledgements The research carried out within the national group GNAMPA of INdAM.

The first and fourth author have been supported by the INdAM-GNAMPA Project 2016 "Metodi topologici, sistemi dinamici e applicazioni".

The second and fourth author have been supported by the project Fondi Ricerca di Base 2016 "Problemi Differenziali Soggetti ad Impulsi Fissi o Variabili", Department of Mathematics and Computer Science, University of Perugia.

The first author has been supported by the project Fondi Ricerca di Base 2016 "Metodi topologici per equazioni differenziali in spazi astratti", Department of Mathematics and Computer Science, University of Perugia.

The third author has been supported by the Polish NCN grant no. 2013/09/B/ST1/01963.

Open Access This article is distributed under the terms of the Creative Commons Attribution 4.0 International License (http://creativecommons.org/licenses/by/4.0/), which permits unrestricted use, distribution, and reproduction in any medium, provided you give appropriate credit to the original author(s) and the source, provide a link to the Creative Commons license, and indicate if changes were made.

\section{Appendix}

For the reader's convenience in this section we collect some of definitions and results the proofs are based on.

In order to consider in Theorems 3.2 and 3.4 a lower semicontinuous map $p$ we need the following approximation result contained in [15].

Lemma A.1 ([15], Lemma 13.2.3) Let $X$ be a Banach space and let $g: X \rightarrow(-\infty,+\infty]$ a lower semicontinuous function with non-empty effective domain satisfying the following condition: there exist two constants $C>0$ and $r \geq 1$

$$
g(x) \geq-C\left(1+\|x\|^{r}\right) \text { for every } x \in X .
$$

Then there exists a sequence $\left\{g_{n}\right\}_{n}$ of functions $g_{n}: X \rightarrow \mathbb{R}$ such that every $g_{n}$ is Lipschitz continuous on bounded subsets of $X$ and $g_{n} \uparrow g$ pointwise on $X$ as $n$ goes to infinity.

To prove the characterization of the equi-Lyapunov pair for the inclusion (2) (see Definition 1.3) we need to give the following tangency concept introduced by Cârjă and Postolache in [14]. Let $K$ be a subset in $E$ and $\xi \in K$. If $A: D(A) \subseteq E \rightarrow E$ is the infinitesimal generator of a $C_{0}$-semigroup $\{U(t)\}_{t \geq 0}$, a function $f \in L_{l o c}^{1}\left(\mathbb{R}^{+} ; E\right)$ is $A$-tangent to the set $K$ at the point $\xi \in K$ if

$$
\liminf _{h \downarrow 0} \frac{1}{h} \operatorname{dist}\left(U(h) \xi+\int_{0}^{h} U(h-s) f(s) d s ; K\right)=0 .
$$

The class of all $A$-tangent functions to $K$ at $\xi \in K$ is denoted by $\mathscr{F}_{K}^{A}(\xi)$.

Remark A.2 ([14], Remark 3) It is possible to characterize the tangency by means of sequences as follows: $f \in \mathscr{F}_{K}^{A}(\xi)$ if and only if there exist sequences $\left\{h_{n}\right\}_{n} \subset \mathbb{R}^{+}$and $\left\{w_{n}\right\}_{n} \subset X$ with $h_{n} \downarrow 0$ and $w_{n} \rightarrow 0$ such that

$$
U\left(h_{n}\right) \xi+\int_{0}^{h_{n}} U\left(h_{n}-s\right) f(s) d s+h_{n} w_{n} \in K, \text { for every } n \in \mathbb{N} .
$$


Moreover, we recall that a uniqueness function $\omega: \mathbb{R}_{0}^{+} \rightarrow \mathbb{R}_{0}^{+}$is a continuous, nondecreasing function, such that the only $C^{1}$-solution of the Cauchy problem

$$
\left\{\begin{array}{l}
x^{\prime}(t)=\omega(x(t)) \\
x(0)=0
\end{array}\right.
$$

is $x \equiv 0$ (see [14, Definition 4]). Given the Cauchy problem

$$
\left\{\begin{array}{l}
u^{\prime}(t) \in A u(t)+F(u(t)), t \in[0, T] \\
u(0)=\xi \in E
\end{array}\right.
$$

where $A: D(A) \subseteq E \rightarrow E$ is a linear operator satisfying (A), $F: E \multimap E$ is a multimap, a subset $K \subset E$ is said to be invariant with respect to $A+F$ if for every $\xi \in K$ each mild solution $u:[0, T] \rightarrow E$ of the problem (24) is in $K$, that is $u(t) \in K$, for every $t \in[0, T]$. In Section 3 we use the following result.

Theorem A.3 ([14], Theorem 2) Let $E$ be a separable Banach space, $A: D(A) \subseteq E \rightarrow E$ be a linear operator satisfying (A), $K$ a nonempty and closed subset of $E$ and $F: E \multimap E$ a nonempty, closed and bounded valued multimap. Assume that

(a) there exists an open neighborhood $W \subseteq E$ of $K$, such that for every $\xi \in K$ and $\Omega \subseteq W$, a bounded open set containing $\xi$, there exists an uniqueness function $\omega_{\Omega}$ : $\mathbb{R}_{0}^{+} \rightarrow \mathbb{R}_{0}^{+}$such that

$$
F(x) \subset F(y)+\omega_{\Omega}(\|x-y\|) B_{E}^{1}(0),
$$

for every $x \in \Omega \backslash K, y \in K \cap \Omega$;

(b) for every $\xi \in K$,

$$
F(\xi)_{L^{1}} \subset \mathscr{F}_{K}^{A}(\xi)
$$

Then $K$ is invariant with respect to $A+F$.

\section{References}

1. Adly, S., Hantoute, A., Théra, M.: Nonsmooth Lyapunov pairs for infinite-dimensional first-order differential inclusions. Nonlinear Anal. 75(3), 985-1008 (2012)

2. Adly, S., Hantoute, A., Théra, M.: Nonsmooth Lyapunov pairs for differential inclusions governed by operators with nonempty interior domain. Math. Program. 157(2, Ser. B), 349-374 (2016)

3. Akhmet, M.: Principles of Discontinuous Dynamical Systems. Springer, New York (2010)

4. Bainov, D., Covachev, V.: Impulsive Differential Equations with a Small Parameter, volume 24 of Series on Advances in Mathematics for Applied Sciences. World Scientific Publishing Co., Inc., River Edge (1994)

5. Belarbi, A., Benchohra, M.: Existence theory for perturbed impulsive hyperbolic differential inclusions with variable times. J. Math. Anal. Appl. 327(2), 1116-1129 (2007)

6. Benchohra, M., Hedia, B.: Impulsive functional differential inclusions with state-dependent delay and variable times. Commun. Appl. Anal. 16(1), 47-62 (2012)

7. Benchohra, M., Henderson, J., Ntouyas, S.K.: On first order impulsive differential inclusions with periodic boundary conditions. Dyn. Contin. Discrete Impuls. Syst. Ser. A Math. Anal. 9(3), 417-427 (2002). Advances in impulsive differential equations

8. Benedetti, I., Rubbioni, P.: Existence of solutions on compact and non-compact intervals for semilinear impulsive differential inclusions with delay. Topol. Methods Nonlinear Anal. 32(2), 227-245 (2008)

9. Cardinali, T., Rubbioni, P.: On the existence of mild solutions of semilinear evolution differential inclusions. J. Math. Anal. Appl. 308(2), 620-635 (2005)

10. Cârjă, O.: Lyapunov pairs for multi-valued semi-linear evolutions. Nonlinear Anal. 73(10), 3382-3389 (2010) 
11. Cârjă, O., Lazu, A.: Lyapunov pairs for continuous perturbations of nonlinear evolutions. Nonlinear Anal. 71(3-4), 1012-1018 (2009)

12. Cârjă, O., Motreanu, D.: Flow-invariance and Lyapunov pairs. Dyn. Contin. Discrete Impuls. Syst. Ser. A Math. Anal. 13B(suppl.), 185-198 (2006)

13. Cârjă, O., Motreanu, D.: Characterization of Lyapunov pairs in the nonlinear case and applications. Nonlinear Anal. 70(1), 352-363 (2009)

14. Cârjă, O., Postolache, V.: Necessary and sufficient conditions for local invariance for semilinear differential inclusions. Set-Valued Var. Anal. 19(4), 537-554 (2011)

15. Cârjă, O., Necula, M., Vrabie, I.I.: Viability, Invariance and Applications, volume 207 of North-Holland Mathematics Studies. Elsevier Science B.V., Amsterdam (2007)

16. Ergören, H.: Impulsive functional delay differential inclusions of fractional order at variable times. Adv. Difference Equ. 37(13), 2016 (2016)

17. Frigon, M., O'Regan, D.: First order impulsive initial and periodic problems with variable moments. J. Math. Anal. Appl. 233(2), 730-739 (1999)

18. Górniewicz, L., Ntouyas, S.K., O’Regan, D.: Existence results for first and second order semilinear impulsive differential inclusions. Topol. Methods Nonlinear Anal. 26(1), 135-162 (2005)

19. Graef, J.R., Henderson, J., Ouahab, A.: Impulsive Differential Inclusions. A fixed point approach, volume 20 of De Gruyter Series in Nonlinear Analysis and Applications. De Gruyter, Berlin (2013)

20. Grudzka, A., Ruszkowski, S.: Structure of the solution set to differential inclusions with impulses at variable times. Electron. J. Differ. Equ. 114, 16 pp. (2015)

21. Hakl, R., Pinto, M., Tkachenko, V., Trofimchuk, S.: Almost periodic evolution systems with impulse action at state-dependent moments. J. Math. Anal. Appl. 446(1), 1030-1045 (2017)

22. Hu, S., Papageorgiou, N.S.: Handbook of Multivalued Analysis. Applications, vol. II, volume 500 of Mathematics and its Applications. Kluwer Academic Publishers, Dordrecht (2000)

23. Kamenskii, M., Obukhovskii, V., Zecca, P.: Condensing Multivalued Maps and Semilinear Differential Inclusions in Banach Spaces, volume 7 of De Gruyter Series in Nonlinear Analysis and Applications. Walter de Gruyter \& Co., Berlin (2001)

24. Kocan, M., Soravia, P.: Lyapunov functions for infinite-dimensional systems. J. Funct. Anal. 192(2), 342-363 (2002)

25. Lakshmikantham, V., Baĭnov, D.D., Simeonov, P.S.: Theory of Impulsive Differential Equations, volume 6 of Series in Modern Applied Mathematics. World Scientific Publishing Co., Inc., Teaneck (1989) 